\title{
A local reference value for the mental foramen in adult Sudanese: A 3D computerized tomography based study
}

\author{
Khalid Mohammed Ahmed ${ }^{1}$, Caroline Edward Ayad ${ }^{1}$, Samih Awad Kajoak ${ }^{2}$ \\ ${ }^{I}$ College of Medical Radiological Science- Sudan University Of Science And Technology, Khartoum-Sudan \\ ${ }^{2}$ College of Applied Medical Science, Diagnostic Radiology Department, Hail University-Saudi Arabia.
}

\begin{abstract}
The mental foramen $(M F)$ is located on the anterior surface of the mandible. It permits passage of the mental nerve and vessels. Studying the morphological variation of $(M F)$ helps to localize the mental nerve to prevent complications during surgery. $(M F)$ character varied considerably among ethnic groups, although, determination the norms is essential, however no study was conducted in Sudanese. The aims of this study are to establish local reference for $(M F)$ width for Sudanese as well as to study the age and gender related difference and to compare the measurements with other populations.

The present study was carried out in the Radiology department-Military Hospital, Khartoum -Sudan on 160 adult Sudanese with mean age is 35.26years old. Three dimensional computerized tomography was obtained for the facial bone. The Mandibles were observed for the right and left of $(M F)$ width.

In our study we observed that the (MF) mean width were 4.28 and $3.53 \mathrm{~mm}$ for right and left sides. The (MF) differs between the two genders significantly at $p \leq 0.035$ and 0.010 for right and left sides in respectively.A significant changes in $(M F)$ width were detected in different age groups for both right and left sides. New equations were established to identify the (MF) width as local reference for Sudanese population of known age. The present analysis revealed variations in width of MF of Sudanese from other populations. The (MF) width differs according to age and gender.
\end{abstract}

Keywords: Mental foramen, Morphometry, Computerized Tomography

\section{Introduction}

Anatomically, there is one mental foramen (MF) on each side of the mandible through which passes the mental artery, vein and the mental nerves, the larger of the two terminal branches of the inferior alveolar nerve. The number of mental nerves can vary in individuals and in different races. [1,2] The (MF) is a strategically important landmark during osteotomy procedures, anesthetic nerve blocks and prevention of neurovascular complications after invasive procedures on the lower jaw. Its anatomy is important for evaluating the morphometry of the (MF) in different populations.[3] Different studies were obtained regarding the morphometric characteristics of the (MF); in so doing depicting variable racial tendency. [4]In Africans; the (MF) was observed to exhibit dimorphism; the average size of the short axis of the foramen was $3.97 \mathrm{~mm}$ in the male $3.87 \mathrm{~mm}$ in the female mandibles.[5] These measurements were statistically similar to another study on mandibles from Alagoas state.[6].In Asian /Japanese population the (MF) was observed to have largest horizontal diameter ranged between 3.25-3.32mm [6] In Sri Lankans; the mean transverse diameters of the foramen were $3.31 \mathrm{~mm}$ [7] while in Israelis; the (MF) average diameter was of $2.37 \mathrm{~mm}$.[8]In Turkish; the horizontal diameter of the (MF) was $2.93 \mathrm{~mm}$ on the right side and $3.14 \mathrm{~mm}$ on the left side. [6]

Racial variances in mandibular dimensions have been studied among several Caucasian, African, Asian, and Arab populations.[9-12] An important landmark in carrying out the mental or incisive nerve block is the mental foramen [13] The position of this foramen has also been studied, with racial differences again being found [14-19] What is not known, is whether there are significant variations on these mandibular landmarks(MF) in Sudanese patients compared with those already reported. There are no apparent published reports on its relative short axis in Sudanese populations. Although the variations appears according to the ethnic groups, this study aims to measure the MF width and to find out the variations related to age and gender using 3D CT done for adult Sudanese population as well as to compare the findings with other populations and to establish a local reference for Sudanese regarding the MF width.

\subsection{Equipment:}

\section{Materials and Methods}

The equipment required is CT Toshiba Aquilion(64slices) and three dimensional reconstruction system with parameters is used as below: $\mathrm{Kv}=120, \mathrm{~mA}=300$, Time $=0.75 \mathrm{sec}, \mathrm{mAs}=225$,Slice thickness $=3 \mathrm{~mm}$ $5 \mathrm{~mm}$ 


\subsection{Methods of data measurement:}

This is a descriptive analytical study that deals with scanning patients head (brain and facial structures).The study selected 160 patients (males and females) with CT (64slices). Axial projection was applied with brain protocols as well as sagittal views for facial protocols. All of these protocols used two dimensional as localizer and Digital Imaging and Communications in Medicine (DICOM) files were rendered into threedimensional reconstruction using Anatomize Invivo5 software. The width of the mandible foramen right and left were measured.

\subsection{Sample.}

The study population was composed of both genders presenting to the CT unit of Military Hospital in Khartoum during the period from December 2013 to June 2016.The sample size consisted of 160 patients with different age and gender (92males and 68 females )The radiographs that were clinically diagnosed as normal mandible were included. Any abnormality of mandible for example: fractures, infection, tumors and other mandible diseases were excludes

\subsection{Technique:}

The patients were positioned supine on the CT examination table and head is rested on head holder while removing all metallic and jewelry, straps and pillow were used to help in maintaining the correct positioning, the scan started from base of the skull and end inferior to mental protuberance as well as image were produced in axial projection. Three dimensional volume rendering technique was applied to reconstruct the image data of patients (analytical software) uses several types of reconstruction algorithm with different planes

\subsection{Ethical Consideration}

-No identification or individual details were published.

-No information or patient details were disclosed or used for reasons other than the study

\section{Results}

Table 1: shows the classification of age classes presented in frequency and percentages

\begin{tabular}{|c|c|c|}
\hline & Frequency & Percentages (\%) \\
\hline $15-24$ & 39 & 24.4 \\
\hline $25-34$ & 36 & 22.5 \\
\hline $35-44$ & 51 & 31.9 \\
\hline $45-54$ & 23 & 14.4 \\
\hline $55-64$ & 8 & 5.0 \\
\hline $65+$ & 3 & 1.9 \\
\hline Total & 160 & $100 \%$ \\
\hline \multicolumn{2}{|c}{} \\
\hline
\end{tabular}

Table 2: shows the mean and standard deviation of patients ages, Right and left Foramen Width/(MM)

\begin{tabular}{|c|c|c|c|}
\hline & Age/Years & $\begin{array}{c}\text { Right Foramen } \\
\text { Width/(MM) }\end{array}$ & $\begin{array}{c}\text { Left Foramen } \\
\text { Width(MM) }\end{array}$ \\
\hline Mean & 35.2625 & 4.2881 & 3.5338 \\
\hline Std. Deviation & 12.33112 & 2.30409 & .57263 \\
\hline Minimum & 15.00 & 3.10 & 2.40 \\
\hline Maximum & 75.00 & 5.10 & 5.20 \\
\hline
\end{tabular}

Table 3 :Independent Samples Test shows the difference between the genders for both right and left foramen width

\begin{tabular}{|c|c|c|c|c|c|}
\hline \multicolumn{6}{|c|}{ Group Statistics } \\
\hline & Gender & $\mathrm{N}$ & Mean & $\begin{array}{c}\text { Std. } \\
\text { Deviation }\end{array}$ & \multirow{2}{*}{ P-value } \\
\hline \multirow{2}{*}{ Right Foramen Width } & Male & 92 & 4.2348 & .62025 & \multirow{2}{*}{0.035} \\
\cline { 2 - 5 } & Female & 68 & 4.3603 & 3.47375 & \multirow{2}{*}{0.010} \\
\hline \multirow{2}{*}{ Left Foramen Width } & Male & 92 & 3.6337 & .57480 & .54513 \\
\cline { 2 - 5 } & Female & 68 & 3.3985 & & \\
\hline
\end{tabular}


Table 4 . Descriptive Statistics both right and left foramen width classified according to age groups

\begin{tabular}{|c|c|c|c|c|c|c|c|}
\hline & & $\mathbf{N}$ & Mean & $\begin{array}{c}\text { Std. } \\
\text { Deviation }\end{array}$ & Minimum & Maximum & $\begin{array}{c}\mathbf{P}- \\
\text { value }\end{array}$ \\
\hline \multirow{7}{*}{$\begin{array}{c}\text { Right } \\
\text { Foramen } \\
\text { Width }\end{array}$} & $15-24$ & 39 & 4.3718 & .41292 & 3.70 & 5.70 & \multirow[t]{7}{*}{.028} \\
\hline & $25-34$ & 36 & 4.5333 & .67146 & 3.10 & 5.80 & \\
\hline & $35-44$ & 51 & 3.8039 & .38934 & 3.30 & 4.80 & \\
\hline & $45-54$ & 23 & 5.0957 & 5.95792 & 3.20 & 32.40 & \\
\hline & 55-64 & 8 & 3.6500 & .24495 & 3.40 & 4.20 & \\
\hline & $65+$ & 3 & 4.0000 & .10000 & 3.90 & 4.10 & \\
\hline & Total & 160 & 4.2881 & 2.30409 & 3.10 & 32.40 & \\
\hline \multirow{7}{*}{$\begin{array}{c}\text { Left } \\
\text { Foramen } \\
\text { Width }\end{array}$} & $15-24$ & 39 & 3.6308 & .38468 & 3.10 & 4.50 & \multirow[t]{7}{*}{.000} \\
\hline & $25-34$ & 36 & 4.0500 & .75043 & 2.40 & 5.20 & \\
\hline & $35-44$ & 51 & 3.2745 & .38306 & 2.50 & 4.20 & \\
\hline & $45-54$ & 23 & 3.2391 & .35896 & 2.40 & 3.70 & \\
\hline & 55-64 & 8 & 3.2000 & .21381 & 2.90 & 3.60 & \\
\hline & $65+$ & 3 & 3.6333 & .05774 & 3.60 & 3.70 & \\
\hline & Total & 160 & 3.5338 & .57263 & 2.40 & 5.20 & \\
\hline
\end{tabular}

Table 5 . Correlation coefficient between the right mandibuler foramen width with age . Significant at $\mathrm{p} \leq 0.005$

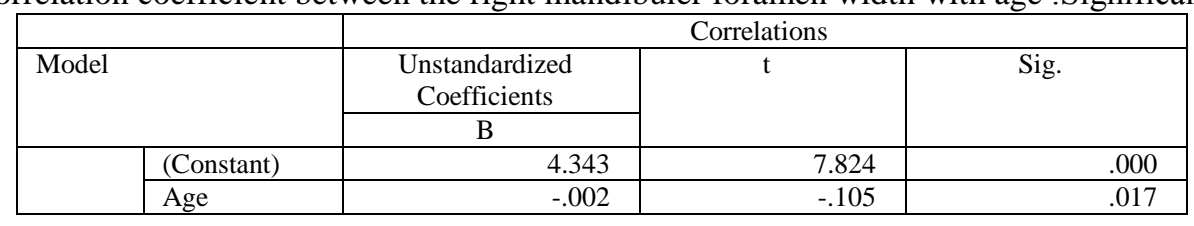

The New Established Equation to predict the right mandibuler foramen width for Sudanese with known age. $\mathbf{R}^{2}=\mathbf{0 . 0 8 4}$

Right mandibuler foramen width= 4.343+(AgeX-0.002)

Table 6 . Correlation coefficient between the left mandibuler foramen width with age . Significant at $p \leq 0.005$

\begin{tabular}{|l|l|r|c|c|}
\hline \multicolumn{2}{|l|}{} & \multicolumn{3}{|c|}{ Correlations } \\
\hline Model & $\begin{array}{c}\text { Unstandardized } \\
\text { Coefficients }\end{array}$ & $\mathrm{t}$ & \multicolumn{2}{|c|}{ Sig. } \\
\cline { 3 - 4 } \multicolumn{2}{|c|}{} & $\mathrm{B}$ & & .000 \\
\hline \multirow{2}{*}{} & (Constant) & 4.025 & 30.583 & .000 \\
\cline { 2 - 4 } & Age & -.014 & -3.953 & \\
\hline
\end{tabular}

The New Established Equation to predict the left mandibuler foramen width for Sudanese with known age. $\mathbf{R}^{2}=0.090$

Left mandibuler foramen width $=4.025+(\operatorname{AgeX}-0.014)$ 


\section{Discussion}

When reviewing the literature regarding morphometric analysis of the MF based on multi-slice computed tomography, no detailed data has been found. The current study provides new data on the width of the MF in a Sudanese population. When practicing dental surgery, orthodontic treatments, maxillofacial trauma, and orthognathic procedures such as a mandibular body osteotomy, in order not to cut or damage the mental nerve, it is important for the operator to know exactly the morphology of MF. [20] Tables 1 and 2 show the classification of age classes presented in frequency and percentages as well as the mean values of the right and left MF width measured for Sudanese.

Radiography is the non-invasive method for diagnosis and treatment planning of major surgical procedures of the mandible. [21]Panoramic radiographs usually are limited due to horizontal overlap of teeth [22] distortion and magnification in orthopantomogram techniques cannot be eliminated, in addition, as the bone density increases, the foramen becomes more difficult to identify and may not be seen clearly. CT has been the first option for diagnosis, surgical planning, and treatment of osseous trauma injuries due to its high specificity and sensitivity.[23] As a result of recent advances in computer hardware and software; 3D imaging by CT scans of craniofacial anomalies is routinely used by most medical centers to define the unique individual aspects of complex anatomy, plan interventions and follow results [24]

Therefore, we utilized CT images because they have certain advantages in diagnostic medicine. In our series of $160 \mathrm{CT}$ images, the width of the MF has been measured; it was found that there is a significant difference between the males and females for both right and left MF width at $p \leq 0.035$ and 0.010 respectively (Table3)Studies have mentioned that the MF morphology, varies not only according to age[25] gender [26,27] and ethnicity; [28,29] but even within the same race; in different geographic regions [30,31,32]and within the inhabitants of the same geographic area[33].

Age difference was also been evaluated and it was found that the age can affected significantly the measurements of MF width (Table 4) .One of the most striking characteristics of the Sudan is the diversity of its people. The Sudanese are divided into multiple ethnic groups and subgroups. Identifying ethnic groups in Sudan was made more complicated by the multifaceted character of internal divisions. Largest ethnic category comprises those considering themselves Arabs, but category internally split by regional and tribal loyalties and affiliation to various groups. Major groups are Nubians and nomadic Beja dwelling in parts of North Africa , and Fur in west. Southern groups include Dinka form the largest portion of the national population (Black), Nuer, and numerous smaller Nilotic and other ethnic groups. Therefore characterization of MF for each tribe is essential. However we characterize MF for Sudanese living in Khartoum State and not considering their origin or tribe which may consider as limitation in our study.

A new equation to predict the right mandibuler foramen width for Sudanese living in central Sudan at the capital (Khartoum) with known age was established.

Right mandibuler foramen width $=4.343+($ AgeX-0.002), R2 $=0.084$. For the left mandibuler foramen width: Left mandibuler foramen width $=4.025+($ AgeX-0.014), $\mathrm{R} 2=0.090$, these are presented in (tables 5 and 6).

The measurements of Sudanese differ from other populations. Data from various ethnic groups including Tanzanian, Thai, Chinese, British, Saudi Arabian vary regarding the characterization of MF.A review by Green (1987) [18] demonstrated a clear racial trend in the characterization of the MF [34] According to Mbajiorgu et al. (1998) [15], in 32 mandibles of black adults from Zimbabwe had mentioned that MF was either round or oval. We suggest that this may be a factor that may change the MF width. Oliveira Junior et al. (2009) [6] reported that the shape was found to be oval with Larger diameter in the horizontal direction. Regarding the size of the mental foramen and according to Chung et al.(1995)[35] ; horizontal opening of MF was $2.4 \mathrm{~mm}$ and Apinhasmit et al.[36] reported the average horizontal opening was 2.8mm. Oguz \& Bozkir (2002) [37] did measurements in 34 dry mandibles of people from Turkey. The horizontal dimension of MF was $2.93 \mathrm{~mm}$ on right side and $3.14 \mathrm{~mm}$ on left side. Souaga et al. [26] studied 61 dry mandibles. The average sizes of the short axes of foramina were $3.97 \mathrm{~mm}$ for males while dimensions of female mandibles was $3.87 \mathrm{~mm}$. The present observations brought out average horizontal dimension of MF to be $4.29 \mathrm{~mm}$ on right side and $3.53 \mathrm{~mm}$ on the left side. This was considered to be greater than other previously studied populations.

\section{Conclusion}

The present analysis revealed variations in width of MF of Sudanese from other populations. The MF width differ according to age and gender 


\section{References}

[1]. Gershenson A, Nathan H, Luchansky E. Mental foramen and mental nerve changes with age. Acta Anat 1986; $126: 21 \pm 28$

[2]. Moiseiwtsch JRD. Position of the mental foramen in a North American, white population. Oral Surg Oral Med Oral Path Oral Radiol Endod 1998; 85: 457 - 460.

[3]. Ahmet Ercan Sekerci, Halil Sahman, Yildiray Sisman, Yusuf Aksu, Morphometric analysis of the mental foramenin a Turkish population based on multi-slicecomputed tomography Journal of Oral and Maxillofacial Radiology / January-April 2013 / Vol 1 | Issue 1,2-7

[4]. Santini A, Land M.A. Comparison of the position of the mental foramen in Chinese and British mandibles. Acta.Anat. 1990;137:208-212.

[5]. Souaga K, Adou A, Angoh Y. Topographical and morphological study of the mandibular foramen in black Africans from the Ivory Coast. Odontostomatol. Trop. $2004 ; 27(105): 17-21$

[6]. Oliveria J E M,. Arajo, A. L. D, SilvaC. M. F,Sousa R C F, Lima FJC. Morphologicaland morphometric study of the mental foramen on the M-CP-18 jiachenjiang point. Int. J. Morphol. 2009; 27(1):231-238.

[7]. Ilayperuma I, Nanayakkara G, Palahepitiya N. Morphometric analysis of the mental foramen in adult Sri Lankan mandibles. Int. J. Morphol.2009; 27(4):1019-1024

[8]. A. Gershenson H, Nathan E, Luchansky. Mental Foramen and Mental Nerve: Changes with Age Acta Anatomica.1986;126:21-28

[9]. Nicholson ML. A study of the position of the mandibular foramen in the adult human mandible. Anat Rec. 1985 May;212(1):110-2.

[10]. Hetson G, Share J, Frommer J, Kronman JH. Statistical evaluation of the position of the mandibular foramen. Oral Surg Oral Med Oral Pathol. 1988 Jan;65(1):32-4.

[11]. Hayward J, Richardson ER, Malhotra SK. The mandibular foramen: its anteroposterior position. Oral Surg Oral Med Oral Pathol. 1977 Dec;44(6):837-43.

[12]. Keros-Naglic J, Panduric J, Buntak-Kobler D. Some anatomical and anthropological measures of mandibular ramus in our population. Coll Antropol. 1997 Jun;21(1):203-10.

[13]. Malamed SF. Handbook of local anesthesia. 4th ed. St Louis: Mosby; 1997

[14]. Moiseiwitsch JR. Position of the mental foramen in a North American, white population. Oral Surg Oral Med Oral Pathol Oral Radiol Endod. 1998 Apr;85(4):457-60.

[15]. Mbajiorgu EF, Mawera G, Asala SA, Zivanovic S. Position of the mental foramen in adult black Zimbabwean mandibles: a clinical anatomical study. Cent Afr J Med. 1998 Feb;44(2):24-30.

[16]. Mwaniki DL, Hassanali J. The position of mandibular and mental foramina in Kenyan African mandibles. East Afr Med J. 1992 Apr;69(4):210-3.

[17]. Shankl and WE. The position of the mental foramen in Asian Indians. J Oral Implantol. 1994;20(2):118-23.

[18]. Green RM. The position of the mental foramen: a comparison between the southern (Hong Kong) Chinese and other ethnic and racial groups. Oral Surg Oral Med Oral Pathol. 1987 Mar;63(3):287-90.

[19]. Al-Khateeb TL, Odukoya O, el-Hadidy MA. Panoramic radiographic study of mental foramen locations in Saudi Arabians. Afr Dent J. 1994;8:16-9.

[20]. Neo J. Position of the mental foramen in Singaporean Malays and Indians. Anesth Prog 1989;36:276-8

[21]. Kaffe I, Ardekian L, Gelerenter I, Taicher S. Location of the mandibular foramen in panoramic radiographs. Oral Surg Oral Med Oral Pathol 1994;78:662-9. 22

[22]. Yosue T, Brooks SL. The appearance of mental foramina on panoramic radiographs. I. Evaluation of patients. Oral Surg Oral Med Oral Pathol 1989;68:360-4.

[23]. Utumi ER, Perrella A, Albuquerque MA, Adde CA, Rocha RG, Cavalcanti MG. Evaluation of simulated bone lesion in the head of the mandible by using multislice computed tomography. J Appl Oral Sci 2009;17:521-6.

[24]. Cavalcanti MG, Vannier MW. Quantitative analysis of spiral computed tomography for craniofacial clinical applications. Dentomaxillofac Radiol 1998;27:344-50.

[25]. A. Gershenson H, Nathan E, Luchansky. Mental Foramen and Mental Nerve: Changes with Age Acta Anatomica.1986;126:21-28

[26]. Souaga K, Adou A, Angoh Y. Topographical and morphological study of the mandibular foramen in black Africans from the Ivory Coast. Odontostomatol. Trop. 2004 ;27(105):17-21.

[27]. Danny R. Sawyer, Michael L. Kielyand Marsha A. Pyle The frequency of accessory mental foramina in four ethnic groups Archives of Oral Biology 1998; 43:417-420

[28]. Zivanovic S. Some morphological characters of the East African mandible. Acta. Anat.1970; 77:109-119.

[29]. Santini A, Land M.A. Comparison of the position of the mental foramen in Chinese and British mandibles. Acta.Anat. 1990;137:208-212.

[30]. Phillips JL, Weller RN, Kulild JC. The mental foramen: Part II. Radiographic position in relation to mandibular second premolar. J of Endodontics 1992; 18: 271-274.

[31]. Weller RN,Phillips JL., Kulild JC. The mental foramen: Part III. Size and position on panoramic radiograph. J of Endodontics 1992; 16: 383-386.

[32]. Lindh C, Petersson A, Kling B. Measurements of distances related to the mandibular canal in radiographs. Clin Oral implant Res. $1995 ; 6: 96-103$.

[33]. Ari I Kafa IM, Basar Z, Kurt M A. The localization and anthropometry of mental foramen on late Byzantine mandibles.Coll. Antropol. 2005;29:233-236.

[34]. Estudio de la Posición, Forma, Tamaño e Incidencia del Foramen Mentoniano y Foramen Mentoniano Accesorio en Cráneos Humanos de Indios Adultos Study of Position, Shape, Size and Incidence of Mental Foramen and Accessory Mental Foramen in Indian Adult Human Skulls Int. J. Morphol., 28(4):1141-1146, 2010.

[35]. Chung, M. S.; Kim, H. J.; Kang, H. S. \& Chung, I. H. Locational relationship of the supraorbital notch or foramen and infraorbital and mental foramina in Koreans.Acta Anat. (Basel), 154:162-6, 1995

[36]. Apinhasmit, W.; Methathrathip, D.; Chompoopong, S. \& Sangvichien, S. Mental foramen in Thais: an anatomical variation related to gender and side. Surg. Radiol. Anat., 28:529-33, 2006.

[37]. Oguz, O. \& Bozkir, M. G. Evaluation of location of mandibular and mental foramina in dry, young, adult human male, dentulous mandibles. West Indian Med. J., 51:14-6,2002. 